\title{
A petrographic thin sectioning technique for evaluating composite materials
}

\author{
D. S. PARKER, A. F. YEE \\ Department of Materials Science and Engineering, University of Michigan, Ann Arbor, Michigan 48105, \\ USA
}

The development of tough high-peformance composites often employs a variety of analytical methods that correlate morphological, yield and fracture characteristics to the corresponding properties of a composite. Most of these techniques can be severely limited due to the high concentration of fibres used in high performance composites (nominally $60 \mathrm{wt} \%$ ).

Several light microscopy techniques can be used to identify relationships between morphological features and composite properties, for example fracture surface studies using reflected light microscopy, microtomed sections, cast films and etched surfaces. These techniques are limited for the following reasons: reflected light microscopy is restricted to surfaces, cast films do not necessarily reflect properties of the bulk material, microtomed sections are very difficult to produce with such a high concentration of fibres, and etched surfaces can be difficult to interpret because artefacts may be created by the use of the etchant.

Petrographic thin sectioning has been used in conjunction with transmission polarized light microscopy for microstructural and deformation mechanism characterizations in a variety of polymer systems such as: identifying the failure modes in glass-filled polycarbonate and toughened nylon [1], evaluating the toughening mechanisms in rubber-toughened epoxy [2], and observing the development of the plastic zone in front of a crack tip in toughened polycarbonates and epoxy resins [3]. The technique used for preparing petrographic thin sections is described by Holik et al. [1] This paper will focus on adaptations of this technique to facilitate the examination of high performance composites.

We have adapted a transmitted light microscopy thin sectioning technique for use in studying high performance composites which overcomes several of the limitations associated with the more standard techniques. Thin sectioning of composites is extremely useful for identifying the presence of spherulites within a composite matrix and relating the effect of thermal treatment on spherulite morphology. This technique is also applicable to subsurface deformation analysis for identifying the plastic zone within a high performance composite. In addition, thin sectioning provides insight into the types of energy absorption mechanisms which occur during fracture. Three types of thermoplastic composite were studied for illustrative purposes: a poly(ether ether ketone) (PEEK)-based, a BPA polycarbonate (PC)-based and a rubber-modified PC-based composite.

Before we discuss the results, a description of our thin sectioning technique for composite materials is in order. First, a composite sample is sectioned perpendicular to the fibre direction using a Buehler Isomet low speed diamond saw. Attempts were made to produce thin sections by sectioning parallel to the fibres; however, once the thin section approached the final thickness, portions of the fibres pulled out of the matrix and damaged the section. This resulted in a surface which could not be successfully polished and therefore was discontinued. Perhaps sectioning slightly off-axis to the fibre direction would provide a successful thin section method; however, this was not investigated. After the sample is sectioned, it is mounted using a two-part epoxy (epoxide resin and hardener, part nos CM-161-R and CM-161-H; Mager Scientific Inc., Dexter, Michigan). Once the mount has cured, the specimen is rough-ground using a standard waterrinse metallographic grinding wheel and silicon carbide abrasives. Alumina powder abrasive was used for fine polishing starting with $5 \mu \mathrm{m}$ and followed by 1.0 , 0.3 and $0.05 \mu \mathrm{m}$. Due to the difference in hardness between the fibres and the matrix, one must be careful to avoid relief polishing. A short nap polishing cloth as well as a minimum polishing time reduces the amount of refief that may occur.

The next step involves mounting the sample on to a glass slide. First the slide is cleaned with isopropyl alcohol and allowed to dry. The slide is then coated with a thin layer of an epoxy adhesive. Adhesives that bond well to both the glass and the sample surface may be used as long as they are transparent and do not alter the specimen's structure. We have found that a two-part adhesive used for bonding fibre optics works very well for this application (TRA-CON epoxy resin TRA-BOND BA-F113; TRA CON Inc, Medford, Massachusetts). The key to adhering the sample to the glass slide is to obtain a very thin adhesive layer that is free from entrapped air. The entrapped air is removed by pressing the sample (polished side down) on to the glass slide. This also aligns the sample surface parallel to the glass slide. The specimen should be lightly clamped while the adhesive cures.

Once the specimen has been mounted to a glass slide, a Buehler Petro-Thin was used to rough-grind the sample to approximately $90 \mu \mathrm{m}$. The Petro-Thin has a glass slide vacuum attachment which maintains the slide parallel to a sectioning and grinding wheel. This equipment is normally used for producing thin sections of petrographic samples, but we found that it works very well for polymeric materials and produces a thinned specimen with parallel surfaces. Using a 

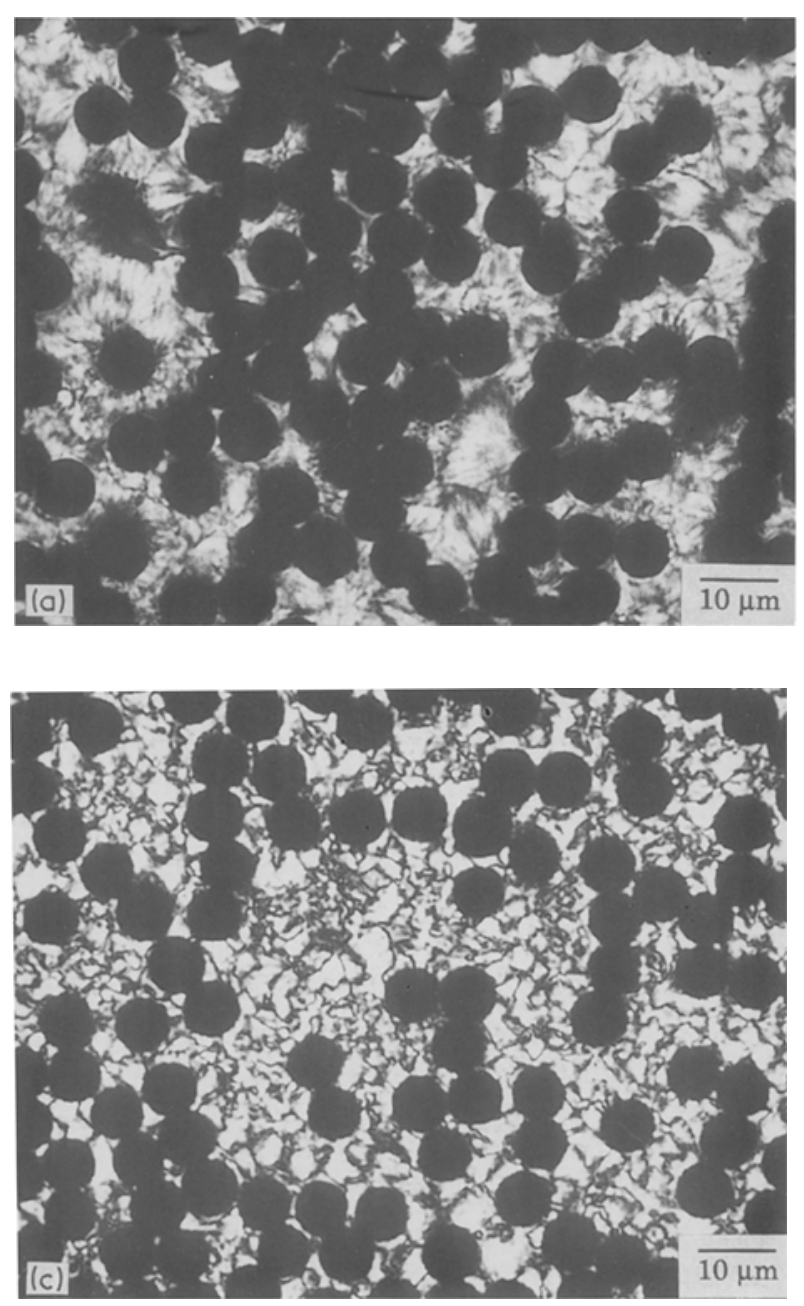

petrographic slide holder and Buehler Ecomet III polishing wheel, the section was thinned to approximately its final thickness using 600 grit silicon carbide paper. The final thickness depends on the type of material and the features one would like to observe. Our samples were thinned to approximately 10 to $20 \mu \mathrm{m}$ as measured with a micrometer. Final polishing using the alumina abrasives in succession completes the specimen preparation. Inspection of the thin sections was done using a Nikon Microphot microscope equipped with two polarizing filters.

The first samples discussed are of a PEEK-AS4

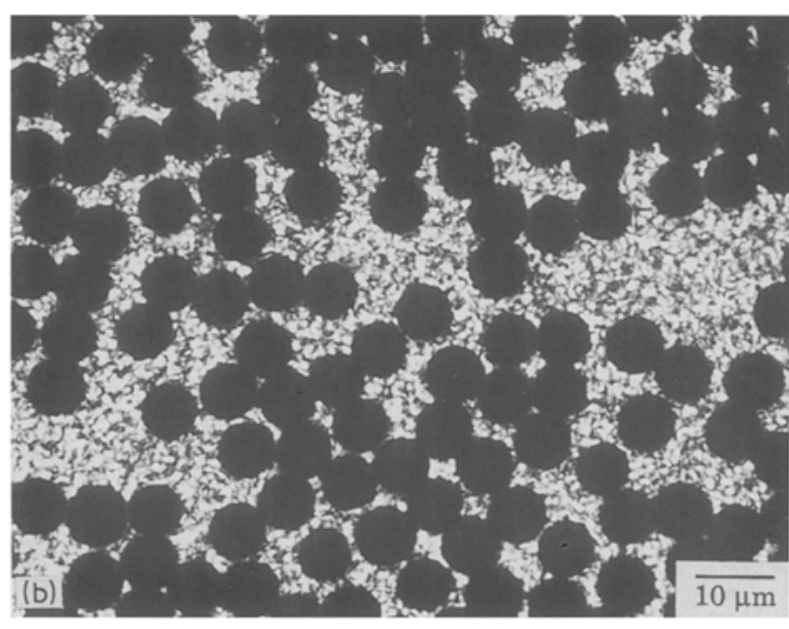

Figure 1 A thin section of a PEEK-AS4 fibre composite illustrating the influence of the cooling rate during molding on the spherulite morphology: (a) $1^{\circ} \mathrm{C} \mathrm{min}^{-1}$, (b) $19^{\circ} \mathrm{Cmin}^{-1}$ and (c) annealed.

unidirectional carbon fibre composite, PEEK is a high performance thermoplastic used in continuous fibre reinforced composites. Upon cooling from the melt, a crystalline structure is formed. The resulting composite properties are affected by the rate at which the polymer is cooled from the melt [4], therefore, it is important to characterize the spherulite morphology. Before our work, a common method for investigating the spherule morphology was by etching the sample with a permanganic etchant, which removes the amorphous regions, and viewing the textured surface using a scanning electron microscope (SEM) [5]. The textured surface is related to the spherulite size. Using an etchant procedure to identify the spherulites may create artefacts such as the preferential removal of material along the fibre-matrix interface and swelling of the matrix. By utilizing the thin sectioning technique described the boundary between spherulites and the fibre-matrix interface is clearly visible and swelling of the matrix should be minimized. PEEK composites were produced with the following thermal treatments: cooled from the melt at 1 and $19^{\circ} \mathrm{C} \mathrm{min}^{-1}$ and annealed at $200^{\circ} \mathrm{C}$ for $24 \mathrm{~h}$. Thin sections of these composites, shown in Fig. 1, illustrate clearly how the spherulitic structure of the matrix changes with thermal

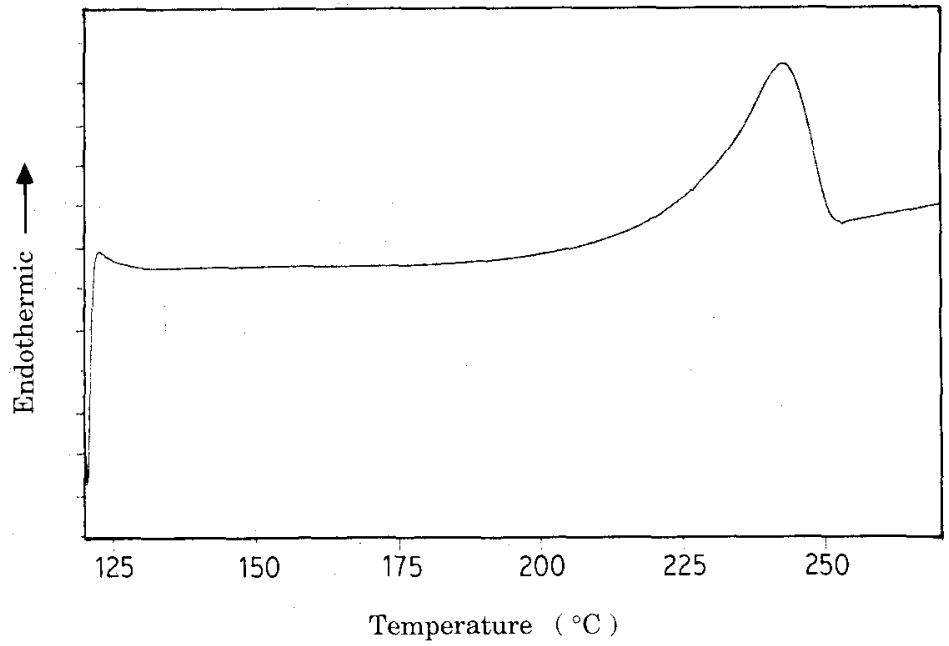

Figure 2 DSC of a solvent-cast film of PC (10 wt \% PC in methylene chloride) indicating that the film is comprised of crystalline material with a melting temperature of $242^{\circ} \mathrm{C}$. The scanning rate was $10^{\circ} \mathrm{C} \mathrm{min}^{-1}$. 


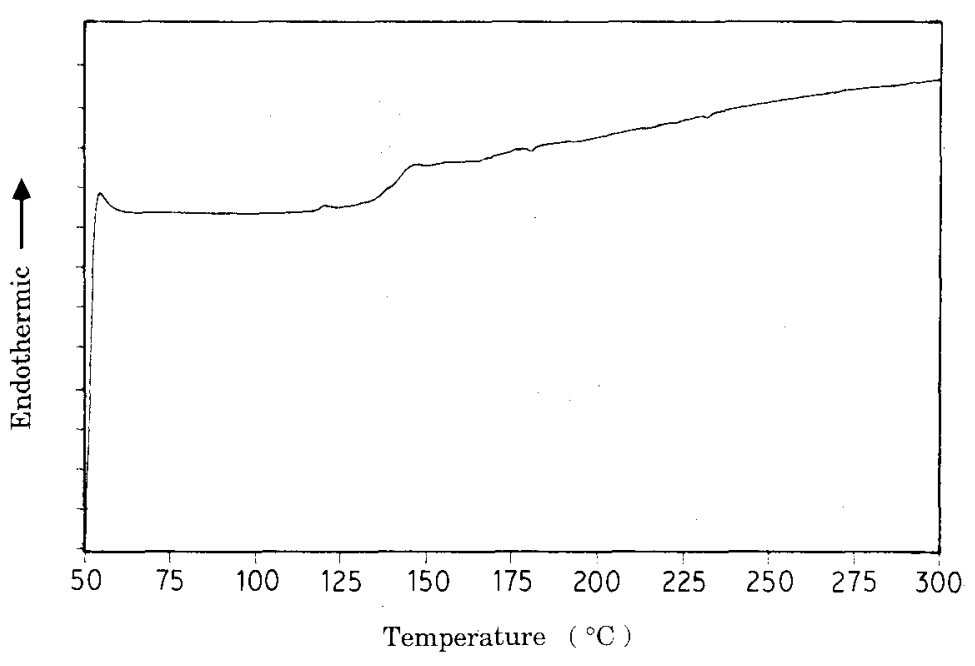

Figure 3 Using a differential scanning calorimeter, no melting point is observed in a polycarbonateAS4 fibre composite. The scanning rate was $10^{\circ} \mathrm{C}$ $\min ^{-1}$.

treatment. It is clear that by using standard stereology techniques, an average spherulite size could be calculated for each thermal treatment and it would also be possible to analyse the nucleation of spherulites along the fibre interface.

Our second example is a polycarbonate-AS4 unidirectional carbon fibre composite. The composite was produced from pre-pregs which were impregnated using a solvent process. Upon removal of the solvent, the matrix becomes crystalline. Without a proper thermal treatment, the crystallinity is carried over into the final composite. The melting temperature for bisphenol A polycarbonate is between 225 and $250^{\circ} \mathrm{C}$ [6]. Using a differential scanning calorimeter (DSC) a melting peak is seen at $242^{\circ} \mathrm{C}$ for a solution-cast sample (which contains no fibres) as shown in Fig. 2. However, in the solvent-impregnated compress-molded composite, where the fibre concentration is $63 \mathrm{wt} \%$, no melting peak is observed as shown in Fig. 3. Therefore, one might conclude that the composite did not contain a significant fraction of crystalline material. However, when a thin section of the same composite is produced and viewed in cross-polarized light, a spherulite morphology is observed as shown in Fig. 4. The reason for the inability of the DSC to detect the presence of crystallinity within the matrix of the composite is unclear. Without producing a thin section,

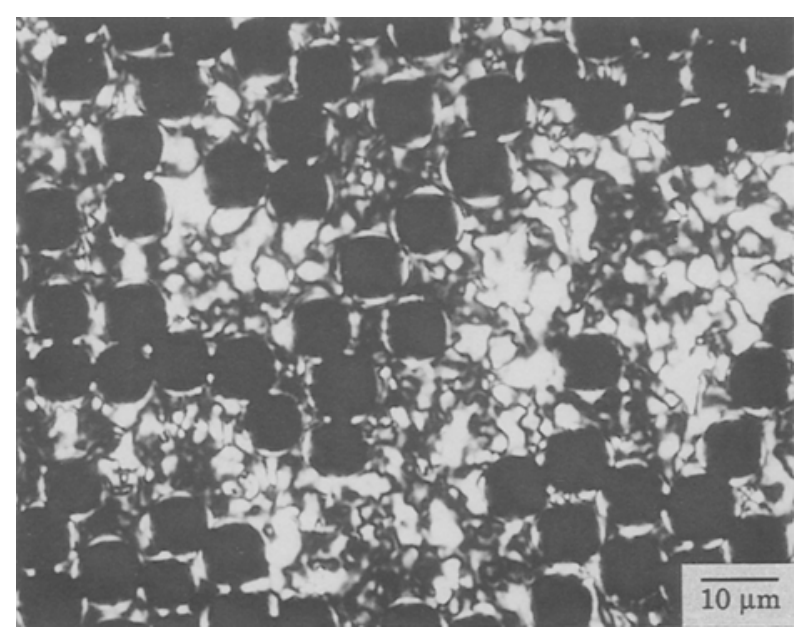

Figure 4 A thin section of the same polycarbonate-AS4 fibre composite analysed using the DSC shown in Fig. 3, indicating the presence of spherulites within the matrix. the assumption that one was studying a composite with an amorphous matrix would have been in error.

The first two examples involved the characterization of the spherulite morphology. The third example will illustrate the usefulness of this technique to study the deformation mechanisms which occur in these complex materials. A rubber-toughened polycarbonate resin was used as a matrix for a continuous AS4 fibre composite (62 wt \% fibre). A double cantilever beam (DCB) specimen was fabricated and tested in Mode I (the crack front moves in a direction parallel to the fibre direction) using a displacement rate of $12.7 \mathrm{~mm} \mathrm{~min}^{-1}$. A thin section was produced of an area where crack arrest had occurred and is shown in Fig. 5. When viewed using cross-polarized light, a shear yielded zone (birefringent region) was clearly observed and extended approximately, 50 to $60 \mu \mathrm{m}$ below the fracture surface. It is commonly believed that the deformation zone will be restricted to the interlaminar region [7]. This would dictate that once the size of the deformation zone of the matrix is equivalent to the interlaminar thickness, the only way further improvements in fracture toughness are possible is by increasing the density of energy absorbed within that region. Thin sectioning provides direct evidence that supports models in which the plastic

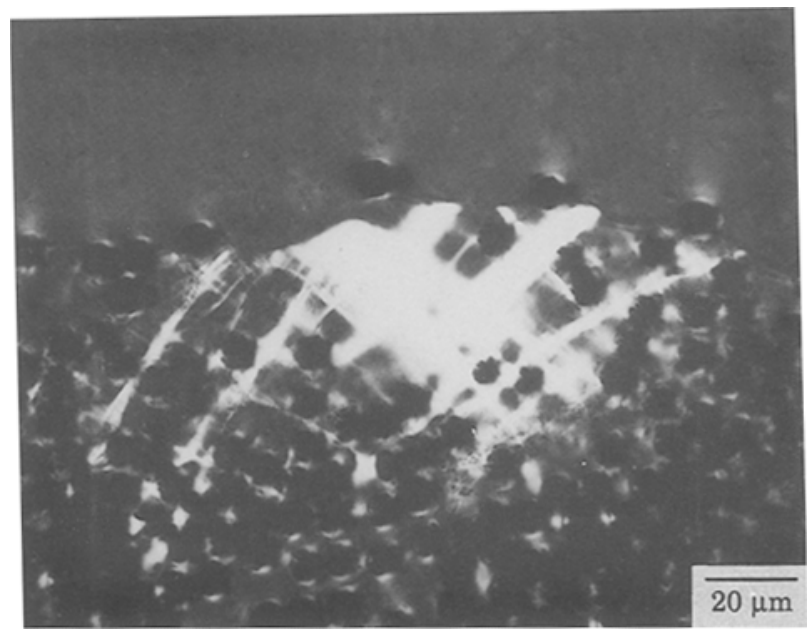

Figure 5 Thin section of a DCB rubber-toughened polycarbonateAS4 fibre composite in the area of crack arrest. A shear-yielded zone is clearly visible which extends well beyond a single interfibrillar spacing. 
zone of a high-performance composite utilizing ductile matrices is not limited to a single interfibrillar spacing [8]. Our work indicates that the constraint of the matrix due to the fibres may not be as great as one would expect. Therefore, if a proper toughening technique for tough matrices is developed which is capable of operating within the high-density fibre regions of a composite, a greater transfer of neat resin toughness improvements to the composite could be possible.

In summary, thin sectioning of high performance composites is very useful for characterizing the matrix morphology and subsurface fracture analysis which would otherwise be impractical utilizing other more standard techniques. Combining the thin sectioning technique described with other standard analysis methods for composites should provide a more complete analysis, and thus aid in producing tough composites.

\section{Acknowledgements}

This work is supported by NASA grant NAG-1-607. We would also like to thank Dr N. Johnston for supplying the polycarbonate composites and Dr I. K. Partridge for supplying the PEEK composites.

\section{References}

1. A. S. HOLIK, R. P. KAMBOUR, D. G. FINK and S. Y. HOBBS, in Proceedings of 11 th Annual Technical Meeting of The International Metallographic Society, "Microstructural Science", Vol. 7, edited by I. Le May, P. A. Fallon and J. L. McCall (Elsevier-North-Holland, 1979) p. 357.

2. R. A. PEARSON and A. F. YEE, J. Mater. Sci. 21 (1986) 2475 .

3. H. J. SUE, R. A. PEARSON, D. S. PARKER, J. HUANG and A. F. YEE, Polym. Prepr. 29 (2) (1988) 147.

4. P. T. CURTIS, P. DAVIES, I, K. PARTRIDGE and J-P. SAINTY, in Proceedings of ICCM VI-ECCM 2, July 1987 (Elsevier Applied Science, London, 1987) p. 4.401.

5. R. A. CRICK, D. C. LEACH, P. J. MEAKIN and D. R. MOORE, J. Mater. Sci. 22 (1987) 2094.

6. J. A. BRYDSON, "Plastic Materials" (Butterworth, London, 1975) p. 453.

7. M. R. PIGGOT, J. Mater. Sci. 23 (1988) 3778.

8. W. L. BRADLEY and R. N. COHEN, ASTM STP 876 (American Society for Testing and Materials, Philadelphia, 1985) p. 389.

\section{Received 16 January}

and accepted 24 February 1989 